\title{
Effect of row spacing and direction of sowing on yield and yield attributing characters of wheat cultivated in Western Chitwan, Nepal
}

\author{
Bisheshwor Prasad Pandey ${ }^{1}$, Komal Bahadur Basnet ${ }^{2}$, Madan Raj Bhatta ${ }^{1}$, \\ Shrawan Kumar Sah ${ }^{2}$, Resham Bahadur Thapa ${ }^{2}$, Tanka Prasad Kandel ${ }^{3^{*}}$ \\ ${ }^{1}$ National Wheat Research Program (NWRP), Rupendehi, Nepal \\ ${ }^{2}$ Institute of Agriculture and Animal Science, Tribhuvan University, Rampur, Nepal \\ ${ }^{3}$ Department of Agroecology, Aarhus University, Research Centre Foulum, Tjele, Denmark; \\ ${ }^{*}$ Corresponding Author: Tanka.Kandel@agrsci.dk
}

Received 17 May 2013; revised 10 June 2013; accepted 17 June 2013

Copyright (C) 2013 Bisheshwor Prasad Pandey et al. This is an open access article distributed under the Creative Commons Attribution License, which permits unrestricted use, distribution, and reproduction in any medium, provided the original work is properly cited.

\begin{abstract}
A field experiment was conducted to study the growth and productivity of wheat as affected by row spacing and direction of sowing at Rampur, Chitwan, Nepal during the 2007-2008 wheat growing season. The experiment was carried out in 3-factors factorial randomized complete block design comprising two varieties (Gautam and $B L-2800)$, three row spacings $(15,20$ and $25 \mathrm{~cm})$ and two row directions of sowing (east-west and north-south). The effects of variety and row direction of sowing on grain yield were significant $(p<0.05)$, but the grain yield was not affected by the row spacing treatment. BL-2800 variety produced higher grain yield $\left(3.53 \mathrm{t} \cdot \mathrm{ha}^{-1}\right)$ as compared to Gautam $\left(3.11 \mathrm{t} \cdot \mathrm{ha}^{-1}\right)$. Both wheat varieties yielded about $11 \%$ higher $(p<0.05)$ grain in the north-south sowing as compared to the eastwest sowing.
\end{abstract}

Keywords: Grain Yield; Row Direction; Row Spacing; Triticum Aestivum; Wheat Variety

\section{INTRODUCTION}

Wheat production is one of the economic mainstays in South Asia [1]. Bread wheat (Triticum aestivum L.) is a major food grain in the Eastern Gangetic Plains of South Asia, a region comprising the plains of eastern India, southern Nepal, and Bangladesh [2]. In Nepal, wheat ranks the third position after rice and maize, in terms of both area and production. Also, wheat is the third most impor- tant APP (Agriculture Perspective Plan) prioritized cereal crop after rice and maize. Its production and productivity in Nepal are 1.39 million metric tons and $2.15 \mathrm{t} \cdot \mathrm{ha}^{-1}$, respectively [3].

Plant stand design is a key parameter for grain yield of wheat and other row crops. Plant stand design affects many factors such as light, water, nutrients, and weeds which are crucial for crop production [4]. Row spacing requirements of wheat depend on architecture and growth pattern of the varieties. For higher yield, higher proportion of incident radiation at the soil surface must be intercepted by crop canopy [5]. If a row distance is too wide, solar radiation that falls between crop rows remains unutilized. On the other hand, plants become crowded and they suffer from mutual shading if the row distance is too narrow. Moreover, yield may be reduced in narrow spacing due to increased competition of plants for nutrient and moisture [6]. Similarly, orientation of the rows also affects photosynthetic efficiency and canopy temperature as it affects interception of solar radiation by the crop canopy [7]. A uniform distribution and proper orientation of plants over a cropped area are needed for greater light interception throughout the crop profile and maximum photosynthetic efficiency by all the leaves of a plant [8].

Traditionally, wheat seeds are broadcasted on the prepared surface in Nepal. Soil is prepared mainly by animal power in the mountains, but use of tractors has become a common practice in the southern plain which is the main region of wheat production. Poor seed bed preparation and manual seed broadcasting have been identified as major causes of lower wheat productivity in Nepal. Mechanizations of agriculture have increased in recent decades and adoption of line sowing by farmers is 
expected in near future. However, there is still a lack of knowledge on proper row-spacing and row direction required for maximal yield of wheat varieties. We, therefore, designed an experiment to examine the effect of row spacing and row-direction on yield and yield attributing characters and grain yield of two wheat varieties.

\section{MATERIALS AND METHODS}

\subsection{Site Characteristics}

The experiment was conducted at the experimental station of Institute of agriculture and Animal Sciences (IAAS), Rampur, Chitwan, Nepal $\left(27^{\circ} 39^{\prime} \mathrm{N}, 84^{\circ} 19^{\prime} \mathrm{E}\right.$, and $190 \mathrm{~m}$ above sea level) during a wheat growing season (November 2007 to April 2008). The average annual precipitation of the experimental site is about $2500 \mathrm{~mm}$ with $74 \%$ falling in summer (from June to September). Winter season is generally dry with occasional rainfall and generally remains foggy with minimum sunshine hours. In winter wheat growing season (October-May), the average precipitation is only about $74 \mathrm{~mm}$ [9]. Monthly average data on maximum and minimum temperatures, total rainfall, relative humidity and sunshine hours during the experimental period of the experimental site are presented in Table 1.

\subsection{Experimental Layout and Management}

The experiment was carried out in 3-factors factorial randomized complete block design comprising two varieties (Gautam and BL-2800), three row spacings (15, 20 and $25 \mathrm{~cm}$ ) and two row directions of sowing (eastwest and north-south) resulting in 12 treatment combinations. Each treatment had three replicated plots with a size of $3 \times 3 \mathrm{~m}$. Seeding was done manually in continuous rows at a rate of $120 \mathrm{~kg} \cdot \mathrm{ha}^{-1}$ for all treatments on 16 November 2007. The numbers of rows per plot were 20, 15 and 12 in 15,20 and $25 \mathrm{~cm}$ row spacings, respectively. A $0.25 \mathrm{~m}$ length on either side of the plots was discarded as border, thus the net plot area was $7.6 \mathrm{~m}^{2}$. The net plot consisted of 7, 5 and 4 central rows in 15,20 and $25 \mathrm{~cm}$ row spacings, respectively. The net plot rows were further guarded by one more row from both sides. The plots were irrigated once 21 days after sowing at crown root initiation stage (CRI). The experimental plots were fertilized with 100:50:25 kg N:P:K ha ${ }^{-1}$ through urea, diammonium phosphate and muriate of potash. Half of the nitrogen and along with the entire quantity of phosphorus and potassium were applied at the time of sowing. The remaining half of the nitrogen was applied first irrigation as top dressing.

\subsection{Soil Properties}

Soil samples were taken randomly from three different spots of each plot at a depth of $0-15 \mathrm{~cm}$ using tube auger to record the initial physico-chemical properties of soil in the experimental site. The soil samples were air dried, grounded and sieved through $2 \mathrm{~mm}$ sieve before analysis. Total nitrogen was determined by Macro-Kjeldhal Method [10], available phosphorus by Olsen's method [11] and available potassium by Flame Photometer method. Organic matter was determined by Walkey and Black method [12], $\mathrm{pH}$ by the method recommended by Pradhan [13] and soil texture by hydrometer method.

\subsection{Light Intensity}

Light intensity was measured with Photometer Model Luxomet 300 (M/S Research Instrumentation, New Delhi, India) at bottom, middle and top $(10 \mathrm{~cm}$ below the topmost point) of crop canopy at three different random spots of each plot from jointing to physiological maturity stage. Light intensity was always measured between 12:00 to 13:00 under the sunny sky conditions.

\subsection{Canopy Temperature}

Canopy temperature was measured simultaneously with light measurement with an infra-red thermometer at bottom, middle and top $(10 \mathrm{~cm}$ below the topmost point) of crop canopy at three different random spots of each plot from heading to physiological maturity stage.

\subsection{Measurements of Plant Growth and Yield}

Twenty uniform plants were selected and demarked in the third row of each plot to record plant height devel-

Table 1. Weather conditions during the experimental period (November 2007-April 2008).

\begin{tabular}{ccccc}
\hline Months & Maximum Temp $\left({ }^{\circ} \mathrm{C}\right)$ & Minimum Temp $\left({ }^{\circ} \mathrm{C}\right)$ & Total Rainfall $(\mathrm{mm})$ & Relative Humidity $(\%)$ \\
\hline Nov & 29.69 & 13.86 & 0.15 & 92.13 \\
Dec & 24.42 & 8.93 & 0.00 & 99.65 \\
Jan & 22.25 & 8.48 & 5.70 & 9.44 \\
Feb & 24.34 & 7.66 & 0.57 & 4.28 \\
Mar & 31.42 & 14.84 & 8.45 & 96.24 \\
Apr & 35.81 & 18.86 & 8.08 & 81.97 \\
\hline
\end{tabular}


opment. Plant height was measured after 15 days of sowing at an interval of 15 days to physiological maturity stage. Prior to harvest, effective tillers (tillers with panicles) from an area of $2.5 \mathrm{~m}^{2}$ were counted in all plots. The crop from net plot area was harvested manually using sickles when the plants turned into yellow, flag leaf dried and kernels obtained their natural color. After harvest, the plants were sun-dried, and plot yield of both grain and straw and 1000 seed weights were determined. Grain yields were adjusted to $12 \%$ moisture after drying the sample at $70^{\circ} \mathrm{C}$ to constant weight.

\subsection{Statistical Analysis}

Measurements from each plot were averaged before statistical analysis. Analysis of variance (ANOVA) was carried out on the data to determine the effect of variety, row distance and row orientation for various measurements using MSTAT-C program (version 2.0, Michigan State University, Michigan, USA). Mean separation was done with Fisher's LSD method at the 5\% level of significance.

\section{RESULTS AND DISCUSSIONS}

\subsection{Soil Properties}

The physico-chemical properties of soil at the experimental site are presented in Table 2. The soil was a loamy sand $(81.2,13.2$ and $5.3 \mathrm{~g} \%$ sand, silt and clay, respectively), slightly acidic ( $\mathrm{pH} 8.7,1: 1$ soil:water) containing $0.17 \%$ total $\mathrm{N}$ and $2.2 \%$ organic carbon. Similarly, the soil had $72 \mathrm{~kg} \cdot \mathrm{ha}^{-1}$ available phosphorous and 108 $\mathrm{kg} \cdot \mathrm{ha}^{-1}$ available potassium.

\subsection{Light Distribution in Canopy}

Gautam had significantly higher light intensity in the bottom level of the plants at jointing, heading and dough

Table 2. Physico-chemical characterization of the soil.

\begin{tabular}{lc}
\hline Properties & Content \\
\hline 1. Physical properties & 81.21 \\
Sand\% & 13.21 \\
Silt $\%$ & 5.33 \\
Clay\% & \\
2. Chemical properties & 5.82 \\
Soil pH & 2.20 \\
Soil organic matter $(\%)$ & 0.17 \\
Total nitrogen $(\%)$ & 72 \\
Available phosphorous $\left(\mathrm{kg} \cdot \mathrm{ha}^{-1}\right)$ & 108 \\
Available potassium $\left(\mathrm{kg} \cdot \mathrm{ha}{ }^{-1}\right)$ & Loamy Sand \\
\hline
\end{tabular}

stages than BL-2800 (Table 3). Moreover, the light intensity measured in the middle level of the plants at dough stage was also significantly higher in Gautam than BL-2800 but opposite result was observed at milking stage. At physiological maturity stage there was no significant different between varieties at any levels of the plants.

The effect of row spacings on light intensity measured at different levels of the plants was non-significant at all growth stages. The effect of row directions was non-significant from jointing to the milking stage. Significantly higher light intensity was recorded in north-south row direction at dough (in the top level) and physiological maturity stages (in the middle and top levels).

\subsection{Canopy Temperature}

In general, canopy temperature was higher at bottom level and then declined in middle and top levels of the crop canopy at reproductive stages (Table 4). This might be due to absorption of the short wave radiation at the top and middle of the crop canopy and the transmission of infra-red radiation to the bottom [14].

The canopy temperature of Gautam was significantly higher at all levels than BL-2800. Such trend was more pronounced at the top level of dough and physiological maturity stages. The higher canopy temperature recorded with Gautam might be due to poor air circulation within the crop canopy [14] as it had higher dry matter accumulation and higher fresh weed biomass.

Row spacings did not cause significant variations in canopy temperature at any level of crop canopy which might be due to same seed rate and lack of rainfall as well as irrigation facilities during the active growth period of the crop. Row directions of sowing showed a significant difference in canopy temperature at all reproductive stages. The canopy temperature recorded with the east-west direction was significantly higher at all levels of different reproductive stages.

\subsection{Plant Height Increment}

Plant height is an important parameter contributing to straw yield which influences the harvest index of the harvested crop. Plant height of wheat varieties was significantly different at various growth stages (Table 5). At the initial stages (15 to 45 DAS) of growth, Gautam was significantly taller than BL-2800. However, BL-2800 was significantly taller than Gautam at the reproductive stages (90 to 120 DAS). The plant height was not affected significantly by row spacing and row direction treatments.

\subsection{Dry Matter Addition}

Dry matter production was affected only by single fa- 
Table 3. Effect of variety, row spacing and direction of sowing on light intensity across the canopy at different phenological stages.

\begin{tabular}{|c|c|c|c|c|c|c|c|c|c|c|c|c|c|c|c|}
\hline \multirow{3}{*}{ Treatments } & \multicolumn{15}{|c|}{ Light Intensity $(\times 000$ Lux $)$} \\
\hline & \multicolumn{3}{|c|}{ Jointing } & \multicolumn{3}{|c|}{ Heading } & \multicolumn{3}{|c|}{ Milking } & \multicolumn{3}{|c|}{ Dought } & \multicolumn{3}{|c|}{ Physiological maturity } \\
\hline & Bot & Mid & Top & Bot & & Top & Bot & Mid & Top & Bot & Mid & Top & Bot & Mid & Top \\
\hline \multicolumn{16}{|c|}{ Variety } \\
\hline Gautam & $6.60^{\mathrm{a}}$ & 11.87 & 17.87 & $4.72^{\mathrm{a}}$ & 12.29 & 31.83 & 6.67 & $18.29^{\mathrm{b}}$ & 35.67 & $19.75^{\mathrm{a}}$ & $41.53^{\mathrm{a}}$ & 53.09 & 23.62 & 44.36 & 56.43 \\
\hline BL 2800 & $4.18^{\mathrm{b}}$ & 10.03 & 16.77 & $3.38^{\mathrm{b}}$ & 11.84 & 33.14 & 5.83 & $22.69^{\mathrm{a}}$ & 37.53 & $14.07^{\mathrm{b}}$ & $34.39^{\mathrm{b}}$ & 52.96 & 22.77 & 44.08 & 55.58 \\
\hline $\mathrm{LSD}_{0.05}$ & 1.36 & $\mathrm{~ns}$ & ns & 1.00 & ns & ns & ns & 4.31 & ns & 4.42 & 5.10 & ns & $\mathrm{ns}$ & $\mathrm{ns}$ & ns \\
\hline \multicolumn{16}{|c|}{ Row Spacing } \\
\hline $15 \mathrm{~cm}$ & 4.49 & 10.50 & 19.30 & 3.98 & 11.77 & 32.43 & 6.70 & 19.12 & 37.28 & 14.99 & 39.73 & 52.78 & 23.56 & 43.58 & 56.88 \\
\hline $20 \mathrm{~cm}$ & 6.17 & 11.49 & 16.46 & 3.78 & 12.14 & 32.21 & 6.04 & 19.55 & 34.63 & 19.45 & 37.21 & 53.19 & 23.84 & 43.62 & 57.00 \\
\hline $25 \mathrm{~cm}$ & 5.51 & 10.85 & 16.21 & 4.38 & 12.29 & 32.83 & 6.01 & 22.80 & 37.90 & 16.28 & 36.95 & 53.10 & 22.58 & 43.69 & 56.95 \\
\hline $\mathrm{LSD}_{0.05}$ & ns & $\mathrm{ns}$ & ns & ns & ns & ns & $\mathrm{ns}$ & $\mathrm{ns}$ & ns & $\mathrm{ns}$ & $\mathrm{ns}$ & ns & $\mathrm{ns}$ & $\mathrm{ns}$ & ns \\
\hline \multicolumn{16}{|c|}{ Row Direction } \\
\hline East-west & 5.00 & 10.08 & 15.94 & 3.85 & 12.30 & 31.64 & 5.95 & 20.36 & 35.57 & 14.84 & 37.54 & $52.01^{\mathrm{b}}$ & 23.09 & $42.50^{\mathrm{b}}$ & $54.69^{\mathrm{b}}$ \\
\hline North-south & 5.78 & 11.81 & 18.71 & 4.24 & 13.12 & 33.33 & 6.55 & 20.62 & 37.63 & 18.97 & 38.38 & $54.04^{\mathrm{a}}$ & 23.30 & $45.98^{\mathrm{a}}$ & $56.72^{\mathrm{a}}$ \\
\hline $\mathrm{LSD}_{0.05}$ & ns & $\mathrm{ns}$ & $\mathrm{ns}$ & $\mathrm{ns}$ & $\mathrm{ns}$ & $\mathrm{ns}$ & $\mathrm{ns}$ & $\mathrm{ns}$ & $\mathrm{ns}$ & $\mathrm{ns}$ & $\mathrm{ns}$ & 1.69 & $\mathrm{~ns}$ & 3.43 & 1.53 \\
\hline Grand mean & 5.39 & 10.94 & 17.32 & 4.19 & 12.25 & 32.48 & 6.25 & 20.49 & 36.60 & 16.90 & 37.96 & 53.02 & 23.25 & 43.97 & 56.32 \\
\hline CV (\%) & 26.56 & 25.95 & 26.57 & 27.87 & 27.37 & 11.09 & 26.58 & 28.47 & 10.65 & 27.82 & 19.45 & 4.62 & 10.36 & 15.41 & 4.78 \\
\hline $\operatorname{SEM}( \pm)$ & 0.56 & 0.97 & 1.57 & 4.19 & 9.53 & 1.04 & 4.79 & 1.80 & 1.12 & 1.84 & 2.13 & 0.70 & 0.69 & 1.43 & 0.64 \\
\hline
\end{tabular}

Different letters in a column indicate significant differences at $p<0.05$.

Table 4. Effect of variety, row spacing and direction of sowing on canopy temperature across the canopy height at different phenological stages of wheat.

\begin{tabular}{|c|c|c|c|c|c|c|c|c|c|c|c|c|}
\hline \multirow{3}{*}{ Treatments } & \multicolumn{12}{|c|}{ Canopy temperature $\left({ }^{\circ} \mathrm{C}\right)$} \\
\hline & \multicolumn{3}{|c|}{ Heading } & \multicolumn{3}{|c|}{ Milking } & \multicolumn{3}{|c|}{ Dough } & \multicolumn{3}{|c|}{ Physiological Maturity } \\
\hline & Bottom & Middle & Top & Bottom & Middle & Top & Bottom & Middle & Top & Bottom & Middle & Top \\
\hline \multicolumn{13}{|c|}{ Variety } \\
\hline Gautam & $21.83^{\mathrm{a}}$ & $21.47^{\mathrm{a}}$ & $20.84^{\mathrm{a}}$ & 26.05 & 24.94 & 23.64 & 32.04 & 30.59 & $32.70^{\mathrm{a}}$ & 31.48 & 31.29 & $31.33^{\circ}$ \\
\hline BL 2800 & $21.11^{\mathrm{b}}$ & $20.77^{\mathrm{b}}$ & $20.01^{\mathrm{b}}$ & 26.40 & 24.57 & 23.59 & 31.55 & 30.26 & $31.64^{\mathrm{b}}$ & 31.20 & 31.17 & $30.27^{\mathrm{b}}$ \\
\hline $\mathrm{LSD}_{0.05}$ & 0.69 & 0.57 & 0.54 & ns & ns & ns & ns & ns & 0.98 & ns & ns & 0.45 \\
\hline \multicolumn{13}{|c|}{ Row Spacing } \\
\hline $15 \mathrm{~cm}$ & 21.69 & 21.28 & 20.69 & 26.48 & 25.27 & 23.89 & 31.97 & 30.69 & 30.45 & $31.40^{\mathrm{ab}}$ & 31.34 & 31.45 \\
\hline $20 \mathrm{~cm}$ & 21.28 & 21.18 & 20.29 & 25.25 & 25.01 & 23.73 & 32.40 & 31.28 & 30.94 & $31.57^{\mathrm{a}}$ & 31.35 & 31.28 \\
\hline $25 \mathrm{~cm}$ & 21.44 & 20.90 & 20.44 & 25.95 & 25.10 & 23.74 & 32.52 & 30.80 & 30.62 & $31.05^{\mathrm{b}}$ & 31.01 & 31.16 \\
\hline $\mathrm{LSD}_{0.05}$ & ns & ns & ns & ns & $\mathrm{ns}$ & ns & ns & ns & ns & 0.48 & ns & ns \\
\hline \multicolumn{13}{|c|}{ Row Direction } \\
\hline East-west & $21.69^{\mathrm{a}}$ & $21.14^{\mathrm{a}}$ & $20.81^{\mathrm{a}}$ & 26.62 & $25.68^{\mathrm{a}}$ & $23.87^{\mathrm{a}}$ & $32.67^{\mathrm{a}}$ & $30.94^{\mathrm{a}}$ & 30.99 & 31.61 & $31.66^{\mathrm{a}}$ & $31.57^{\mathrm{a}}$ \\
\hline North-south & $20.25^{\mathrm{b}}$ & $20.10^{\mathrm{b}}$ & $20.14^{\mathrm{b}}$ & 26.13 & $25.00^{\mathrm{b}}$ & $23.13^{\mathrm{b}}$ & $31.03^{\mathrm{b}}$ & $30.21^{\mathrm{b}}$ & 30.15 & 31.27 & $31.01^{\mathrm{b}}$ & $31.03^{\mathrm{b}}$ \\
\hline $\mathrm{LSD}_{0.05}$ & 0.69 & 0.57 & 0.54 & $\mathrm{~ns}$ & 0.60 & 0.56 & 0.97 & 0.60 & $\mathrm{~ns}$ & $\mathrm{~ns}$ & 0.50 & 0.45 \\
\hline Grand mean & 21.32 & 20.97 & 20.47 & 26.12 & 25.08 & 23.65 & 32.02 & 30.68 & 31.07 & 31.36 & 31.26 & 31.15 \\
\hline CV (\%) & 4.69 & 3.94 & 3.88 & 3.16 & 3.45 & 3.41 & 4.38 & 2.83 & 4.65 & 1.84 & 2.35 & 2.12 \\
\hline $\operatorname{SEM}( \pm)$ & 0.29 & 0.24 & 0.24 & 0.23 & 0.25 & 0.23 & 0.40 & 0.25 & 0.41 & 0.16 & 0.21 & 0.19 \\
\hline
\end{tabular}

Different letters in a column indicate significant differences at $p<0.05$.

ctors like variety, row spacing and row direction of sowing (Table 6). As there was no significant effect of row spacing on dry matter accumulation at most of the growth stages, the grain yield was also not affected significantly. The effect of row direction of sowing on dry mater accumulation was found significant at jointing and milking stages

\subsection{Effective Tillers per Unit Area}

The variety differed significantly in producing productive or effective tillers. BL-2800 had significantly 
Table 5. Effect of variety, row spacing and direction of sowing on plant height of wheat.

\begin{tabular}{|c|c|c|c|c|c|c|c|c|}
\hline \multirow[t]{3}{*}{ Treatments } & \multicolumn{8}{|c|}{ Plant height $(\mathrm{cm})$} \\
\hline & \multicolumn{8}{|c|}{ Days after sowing (DAS) } \\
\hline & 15 & 30 & 45 & 60 & 75 & 90 & 105 & 120 \\
\hline \multicolumn{9}{|l|}{ Variety } \\
\hline Gautam & $15.77^{\mathrm{a}}$ & 28.83 & $44.36^{\mathrm{a}}$ & 64.35 & 78.41 & $86.32^{\mathrm{b}}$ & $90.74^{\mathrm{b}}$ & $92.60^{\mathrm{b}}$ \\
\hline BL 2800 & $14.94^{\mathrm{b}}$ & 28.05 & $40.84^{\mathrm{b}}$ & 61.15 & 78.79 & $96.89^{\mathrm{a}}$ & $97.64^{\mathrm{a}}$ & $100.1^{\mathrm{a}}$ \\
\hline $\mathrm{LSD}_{0.05}$ & 0.71 & $\mathrm{~ns}$ & 3.06 & ns & $\mathrm{ns}$ & 2.70 & 2.40 & 2.44 \\
\hline \multicolumn{9}{|l|}{ Row Spacing } \\
\hline $15 \mathrm{~cm}$ & 14.79 & 28.30 & 42.07 & 61.76 & 77.51 & 90.28 & 92.83 & 95.04 \\
\hline $20 \mathrm{~cm}$ & 15.68 & 28.81 & 43.08 & 63.97 & 79.76 & 92.45 & 95.25 & 97.22 \\
\hline $25 \mathrm{~cm}$ & 15.59 & 28.20 & 42.65 & 62.52 & 78.54 & 92.09 & 94.49 & 96.78 \\
\hline $\operatorname{LSD}_{0.05}$ & $\mathrm{~ns}$ & $\mathrm{~ns}$ & $\mathrm{~ns}$ & $\mathrm{~ns}$ & ns & $\mathrm{ns}$ & $\mathrm{ns}$ & $\mathrm{ns}$ \\
\hline \multicolumn{9}{|l|}{ Row Direction } \\
\hline East-west & 15.46 & 28.74 & 43.19 & 62.98 & 78.56 & 91.43 & 93.39 & 95.71 \\
\hline North-south & 15.25 & 28.13 & 42.00 & 62.52 & 78.65 & 91.79 & 94.99 & 96.99 \\
\hline $\mathrm{LSD}_{0.05}$ & ns & ns & ns & ns & ns & ns & ns & ns \\
\hline Grand mean & 15.35 & 28.43 & 42.59 & 62.75 & 78.60 & 91.60 & 94.19 & 96.34 \\
\hline CV (\%) & 6.73 & 8.94 & 10.41 & 7.74 & 5.72 & 4.28 & 3.71 & 3.68 \\
\hline $\operatorname{SEM}( \pm)$ & 0.298 & 0.733 & 1.28 & 1.40 & 1.29 & 1.13 & 1.01 & 1.02 \\
\hline
\end{tabular}

Different letters in a column indicate significant differences at $p<0.05$.

Table 6. Effect of variety, row spacing and direction of sowing on dry matter accumulation at different phenological stages of wheat.

\begin{tabular}{|c|c|c|c|c|c|}
\hline \multirow[t]{2}{*}{ Treatments } & \multicolumn{5}{|c|}{ Dry matter $\left(\mathrm{g} / 375 \mathrm{~cm}^{2}\right)$} \\
\hline & Maximum Tillering & Jointing & Heading & Milking & Physiol Maturity \\
\hline \multicolumn{6}{|l|}{ Variety } \\
\hline Gautam & 2.97 & 9.75 & 25.55 & $43.33^{\mathrm{a}}$ & $51.71^{\mathrm{a}}$ \\
\hline BL 2800 & 3.01 & 9.94 & 24.65 & $38.67^{\mathrm{b}}$ & $43.20^{\mathrm{b}}$ \\
\hline $\mathrm{LSD}_{0.05}$ & ns & ns & $\mathrm{ns}$ & 4.29 & 5.57 \\
\hline \multicolumn{6}{|l|}{ Spacing } \\
\hline $15 \mathrm{~cm}$ & 2.85 & $7.90^{\mathrm{b}}$ & 24.42 & 38.73 & 49.86 \\
\hline $20 \mathrm{~cm}$ & 3.08 & $11.01^{\mathrm{a}}$ & 25.56 & 41.78 & 45.47 \\
\hline $25 \mathrm{~cm}$ & 3.04 & $10.65^{\mathrm{ab}}$ & 25.31 & 42.50 & 47.04 \\
\hline $\operatorname{LSD}_{0.05}$ & $\mathrm{~ns}$ & 2.76 & $\mathrm{~ns}$ & $\mathrm{~ns}$ & $\mathrm{~ns}$ \\
\hline \multicolumn{6}{|l|}{ Direction } \\
\hline East-west & 3.15 & $11.00^{\mathrm{a}}$ & 25.31 & $43.83^{\mathrm{a}}$ & 48.22 \\
\hline North-south & 2.83 & $8.70^{\mathrm{b}}$ & 24.88 & $38.17^{\mathrm{b}}$ & 46.68 \\
\hline $\mathrm{LSD}_{0.05}$ & ns & 2.25 & $\mathrm{~ns}$ & 4.29 & ns \\
\hline Grand mean & 2.99 & 9.85 & 25.09 & 41.00 & 47.45 \\
\hline CV $(\%)$ & 23.25 & 33.17 & 27.24 & 15.15 & 16.98 \\
\hline $\operatorname{SEM}( \pm)$ & 0.20 & 0.94 & 1.97 & 1.79 & 2.32 \\
\hline
\end{tabular}

Different letters in a column indicate significant differences at $p<0.05$.

higher $(16.55 \%)$ effective tillers as compared to Gautam

(Table 7). Wheat cultivated at $20 \mathrm{~cm}$ row spacing pro- duced significantly more effective tillers as compared to 15 and $25 \mathrm{~cm}$ row spacings. However, there was no sig- 
nificant effect of row-direction.

\subsection{Spike Length and Weight}

Spikes of Gautam were significantly longer (16.18\%) as compared to BL-2800 (Table 7). However, there was no significant effect of row spacing and row direction on spike length. Similarly, there was no significant effect of variety, row spacing, and row-direction on spike weight of wheat.

\subsection{Number of Florets and Grains per Spike}

There was no difference in both varieties in terms of number of florets per spike (Table 7). Narrow row distance $(15 \mathrm{~cm})$ had the lowest number of florets per spike. However, row direction did not have a significant effect. Similarly, number of grains per spike was not affected by the treatments.

\subsection{Kernel Weight}

There was no significant effect of variety, row distance and direction of sowing in kernel weight per spike and 1000 kernel weight (Table 7).

\subsection{Sterility Percentage}

The sterility percentage of Gautam was significantly higher than BL-2800 (Table 7). In wheat, the higher temperature during grain filling period reduces the duration of grain filling and also increases non grain part of wheat ear [15]. This might be the reason for significantly higher sterility percentage in Gautam as compared to BL-2800 as higher temperature was recorded in Gautam canopy during grain filling stages (Table 4). Row spacing had a significant effect on sterility percentage. Row spacing of $25 \mathrm{~cm}$ had significantly higher percentage of sterility compared to other two narrower row spacings. Sterility percentage was not affected by the directions of sowing.

\subsection{Grain Yield}

BL-2800 produced significantly higher $(p<0.05)$ grain yield than Gautam (Table 7). This might be contributed by higher canopy temperatures in canopy towards reproductive phases of the variety as higher temperature reduces the grain yield. However, there was no significant $(p<0.05)$ effect of row spacings on grain yield. Similar insignificant response of row spacing in grain yield and attributing parameters was also observed by [16] when winter wheat was cultivated in $7.5,15,22.5$ and 30 $\mathrm{cm}$ row spacings. Grain yield was significantly higher $(p$ $<0.05$ ) in north-south sowing as compared to east-west sowing. Both wheat varieties yielded about $11 \%$ more grain in north-south sowing as compared to east-west sowing. Thus, significantly lower canopy temperature in reproductive stages (Table 4) as well as relatively higher

Table 7. Yield attributing characters and yield of wheat as affected by variety, row spacing and direction of sowing.

\begin{tabular}{|c|c|c|c|c|c|c|c|c|c|c|c|}
\hline Treatments & $\begin{array}{c}\text { Effective } \\
\text { tillers } / 2.5 \mathrm{~m}^{2}\end{array}$ & $\begin{array}{l}\text { Spike } \\
\text { length } \\
(\mathrm{cm})\end{array}$ & $\begin{array}{l}\text { Spike } \\
\text { wt }(g)\end{array}$ & $\begin{array}{c}\text { No. of } \\
\text { florets/spike }\end{array}$ & $\begin{array}{c}\text { No. of } \\
\text { grains/spike }\end{array}$ & $\begin{array}{c}\text { Grain } \\
\text { wt/Spike (g) }\end{array}$ & $\begin{array}{c}1000 \text { kernel } \\
\text { wt }(\mathrm{g})\end{array}$ & $\begin{array}{c}\text { Sterility } \\
(\%)\end{array}$ & $\begin{array}{c}\text { Straw yield } \\
\left(\mathrm{t} \cdot \mathrm{ha}^{-1}\right)\end{array}$ & $\begin{array}{l}\text { Grain yield } \\
\left(\mathrm{t} \cdot \mathrm{ha}^{-1}\right)\end{array}$ & $\begin{array}{c}\text { Harvest } \\
\text { Index }(\%)\end{array}$ \\
\hline \multicolumn{12}{|l|}{ Variety } \\
\hline Gautam & $608.8^{\mathrm{b}}$ & $8.98^{\mathrm{a}}$ & 2.74 & 45.93 & 40.41 & 2.07 & 52.59 & $12.61^{\mathrm{a}}$ & 6.71 & $3.11^{\mathrm{b}}$ & $31.88^{\mathrm{b}}$ \\
\hline BL-2800 & $709.6^{\mathrm{a}}$ & $7.73^{\mathrm{b}}$ & 2.78 & 43.88 & 39.17 & 1.97 & 52.68 & $9.92^{b}$ & 6.32 & $3.53^{\mathrm{a}}$ & $35.82^{\mathrm{a}}$ \\
\hline \multicolumn{12}{|l|}{ Row Spacing } \\
\hline $15 \mathrm{~cm}$ & $634.0^{\mathrm{b}}$ & 8.48 & 2.84 & $42.89^{\mathrm{b}}$ & 38.65 & 1.96 & 52.96 & $10.81^{\mathrm{ab}}$ & 6.74 & 3.27 & $32.91^{\mathrm{b}}$ \\
\hline $20 \mathrm{~cm}$ & $719.7^{\mathrm{a}}$ & 8.61 & 2.65 & $46.88^{\mathrm{a}}$ & 40.06 & 2.04 & 52.28 & $12.48^{\mathrm{a}}$ & 6.59 & 3.38 & $33.95^{\mathrm{ab}}$ \\
\hline $25 \mathrm{~cm}$ & $623.9^{\mathrm{b}}$ & 7.99 & 2.79 & $44.94^{\mathrm{ab}}$ & 40.66 & 2.07 & 52.08 & $10.52^{b}$ & 6.22 & 3.30 & $34.69^{\mathrm{a}}$ \\
\hline $\mathrm{LSD}_{0.05}$ & 80.35 & $\mathrm{~ns}$ & ns & 3.65 & ns & ns & ns & 1.83 & ns & ns & 1.45 \\
\hline \multicolumn{12}{|l|}{ Row Direction } \\
\hline East-west & 649.8 & 8.50 & 2.85 & 44.46 & 39.35 & 1.99 & 52.08 & 11.08 & $6.10^{\mathrm{b}}$ & $3.15^{b}$ & 34.03 \\
\hline North-south & 668.6 & 8.22 & 2.67 & 45.35 & 40.23 & 2.05 & 52.80 & 11.46 & $6.93^{\mathrm{a}}$ & $3.49^{\mathrm{a}}$ & 33.67 \\
\hline $\mathrm{LSD}_{0.05}$ & ns & ns & ns & ns & ns & ns & ns & ns & 0.51 & 0.20 & ns \\
\hline Grand mean & 659.1 & 8.36 & 2.76 & 44.90 & 39.78 & 2.02 & 52.43 & 11.26 & 6.52 & 3.32 & 33.84 \\
\hline CV (\%) & 14.40 & 9.74 & 22.81 & 9.61 & 9.21 & 9.79 & 2.93 & 19.24 & 11.49 & 8.74 & 5.08 \\
\hline $\operatorname{SEM}( \pm)$ & 27.40 & 0.23 & 0.18 & 1.24 & 1.05 & 0.05 & 0.44 & 0.62 & 0.21 & 0.08 & 0.49 \\
\hline
\end{tabular}

Different letters in a column indicate significant differences at $p<0.05$. 
light intensity (Table 3) might have contributed for higher grain yield in north-south row direction as they influence both respiration and photosynthesis. Wall and Kanemasu [17] also observed that a north-south-oriented wheat canopy had captured light more efficiently compared to an east-west orientation which subsequently increased photosynthesis rate, particularly during high zenith angles on clear days. Such a higher rate of photosynthesis might have achieved in north-south orientation resulting in increased yield.

\subsection{Straw Yield and Harvest Index}

Straw yields from both varieties were rather similar (Table 7). The straw yield declined slightly with the widening of row spacing from 15 to $25 \mathrm{~cm}$ which might be due to less number of rows per plot. However, the straw yield was significantly influenced only by row direction of sowing.

Varieties differed in respect of harvest index. BL-2800 had significantly higher $(p<0.05)$ harvest index as compared to Gautam. Similarly, wheat sown at the widest row spacing $(25 \mathrm{~cm})$ had significantly higher $(p<0.05)$ harvest index than that of narrow row spacings.

\section{CONCLUSION}

Grain yield of BL-2800 was significantly higher which was contributed by more effective tillers and lower sterility percentage. Effect of row distance on grain yield was not significant. Significantly higher grain yield was obtained from north-south row direction which was contributed by cumulative effect of higher number of effective tillers per unit area, grain number per spike and grain weight. Lower canopy temperature and higher incidence of light in top canopy towards crop maturity might have contributed to better photosynthesis in grain fill stage resulting in higher grain yield. The results suggested that BL-2800 performs better in the humid sub-tropical condition of western Chitwan and north-south row direction of sowing produces higher grain yield.

\section{REFERENCES}

[1] Ortiz-Ferrara, G., Joshi, A.K., Chand, R., Bhatta, M.R., Mudwari, A., Thapa, D.B., Suwan, M.A., Saikia, T.P., Chatrath, R., Witcombe, J.R., Virk, D.S. and Sharma, R.C. (2007) Partnering with farmers to accelerate adoption of new technologies in South Asia to improve wheat productivity. Euphytica, 157, 399-407. doi:10.1007/s10681-007-9353-2

[2] Sharma, R.C., Ortiz-Ferrara, G., Crossa, J., Bhatta, M.R., Sufian, M.A., Shoran, J., Joshi, A.K., Chand, R., Singh, G. and Ortiz, R. (2007) Wheat grain yield and stability assessed through regional trials in the Eastern Gangetic Plains of South Asia. Euphytica, 157, 457-464.

\section{doi:10.1007/s10681-007-9470-y}

[3] MOAC (2007) Statistical information on Nepalese agriculture. Government of Nepal, Ministry of Agriculture and Cooperatives, Agribusiness Promotion and Statistics Division, Singha Durbar, Kathmandu, Nepal.

[4] Brant, V., Neckář, K., Pivec, J., Duchoslav, M., Holec, J., Fuksa, P. and Venclová, V. (2009) Competition of some summer catch crops and volunteer cereals in the areas with limited precipitation. Plant, Soil and Environment, 55, 17-24.

[5] Eberbach, P. and Pala, M. (2005) Crop row spacing and its influence on the partitioning of evapotranspiration by winter-grown wheat in Northern Syria. Plant Soil, 268, 195-208. doi:10.1007/s11104-004-0271-y

[6] Das, T.K. and Yaduraju, N.T. (2011) Effects of missingrow sowing supplemented with row spacing and nitrogen on weed competition and growth and yield of wheat. Crop and Pasture Science, 62, 48-57. doi:10.1071/CP10203

[7] Drews, S., Neuhöff, D. and Kopke, U. (2009) Weed suppression ability of three winter wheat varieties at different row spacing under organic farming conditions. Weed Research, 49, 526-533.

doi:10.1111/j.1365-3180.2009.00720.x

[8] Evers, J.B., Huth, N.I. and Renton, M. (2009) Light extinction in spring wheat canopies in relation to crop configuration and solar angle. IEEE Third International Symposium on Plant Growth Modeling, Simulation, Visualization and Applications (PMA), Beijing, 9-13 November 2009, 107-110.

[9] Sharma, R.C. and Duveiller, E. (2004) Effect of helminthosporium leaf blight on performance of timely and lateseeded wheat under optimal and stressed levels of soil fertility and moisture. Field Crops Research, 89, 205-218. doi:10.1016/j.fcr.2004.02.002

[10] Jackson, M.L. (1967) Soil chemical analysis. PrenticeHall, New Delhi

[11] Olsen, S.R., Cole, C.V. and Watanabe, F.S. (1954) Dean estimation of available phosphorus in soils by extraction with sodium bicarbonate. USDA Circular, 939 Gov. Printing Office, Washington DC.

[12] Walkley, A. and Black, I.A. (1934) An examination of the degtjareff method for determining soil organic matter and a proposed modification of the chromic acid titration method. Soil Science, 37, 29-38. doi:10.1097/00010694-193401000-00003

[13] Pradhan, S.B. (2005) Soil and plant analysis manual. Nepal Agricultural Research Council, Nepal.

[14] Sharma, V. and Angiras, N.N. (1996) Effect of row orientations, row spacings and weed control methods on light interception, canopy temperature and productivity of wheat (Triticum aestivum). Indian Journal of Agronomy, 41, 390-396.

[15] Shah, N.H. and Paulsen, G.M. (2003) Interaction of drought and high temperature on photosynthesis and grain-filling of wheat. Plant and Soil, 257, 219-226

[16] Chen, S., Zhang, X., Sun, H., Ren, T. and Wang, Y. (2010) Effects of winter wheat row spacing on evapotranpsira- 
tion, grain yield and water use efficiency. Agricultural Water Management, 97, 1126-1132.

doi:10.1016/j.agwat.2009.09.005

[17] Wall, G.W. and Kanemasu, E.T. (1990) Carbon dioxide exchange rates in wheat canopies. Part I. Influence of canopy geometry on trends in leaf area index, light interception and instantaneous exchange rates. Agricultural and Forest Meteorology, 49, 81-102. doi:10.1016/0168-1923(90)90044-7 